\title{
Bochner-almost periodicity for stochastic processes
}

\author{
Fazia Bedouhene, Omar Mellah ${ }^{\dagger}$ and Paul Raynaud de Fitte ${ }^{\ddagger}$
}

March 4, 2012

\begin{abstract}
We compare several notions of almost periodicity for continuous processes defined on the time interval $I=\mathbb{R}$ or $I=[0,+\infty)$ with values in a separable Banach space $\mathbb{E}$ (or more generally a separable completely regular topological space): almost periodicity in distribution, in probability, in quadratic mean, almost sure almost periodicity, almost equi-almost periodicity. In the deterministic case, all these notions reduce to Bochner-almost periodicity, which is equivalent to Bohr-almost periodicity when $I=\mathbb{R}$, and to asymptotic Bohr-almost periodicity when $I=[0,+\infty)$.
\end{abstract}

\section{Introduction}

Since the creation of the theory of almost periodicity by Harald Bohr in the 1920s, many concepts of almost periodicity have proved useful, in particular those of Stepanov, Besicovitch, and Weyl. In the context of stochastic processes, each of these notions gives rise to several possible definitions, such as: almost periodicity in distribution, in probability, in quadratic mean, almost sure etc (not to mention e.g. processes with almost periodic covariance functions and many others, see [20]). In this paper we study the relationships between some of these definitions for Bochner-almost periodicity. Bochneralmost periodicity coincides with Bohr-almost periodicity when the time

\footnotetext{
*Department of Mathematics Faculty of Sciences University of Tizi-Ouzou, Algeria E-Mail: fbedouhene@yahoo.fr

${ }^{\dagger}$ Laboratoire Raphaël Salem, UMR CNRS 6085, University of Rouen, France and Department of Mathematics Faculty of Sciences University of Tizi-Ouzou, Algeria E-Mail: omellah@yahoo.fr

${ }^{\ddagger}$ Laboratoire Raphaël Salem, UMR CNRS 6085, University of Rouen, France E-Mail: prf@univ-rouen.fr
} 
interval $I$ is the whole line $\mathbb{R}[6]$, but it only amounts to asymptotic Bohralmost periodicity when $I=[0,+\infty)$. It has the advantage over Bohr's definition that it does not depend on the uniform structure of the state space but only on it's topology (see Remark 2.1 in this paper).

Our work completes that of C. Tudor [20] who compared different types of Bohr (or Bochner) almost periodicity for continuous processes in the case when $I=\mathbb{R}$.

As an application of our study, it is possible to compare previous results proving almost periodicity in the Bohr sense of the solution of some stochastic differential equations. For example, in the works of C. Tudor and his collaborators (see in particular $[13,19,1,9]$ ), almost periodicity in distribution of the solutions is proved. On the other hand, in a series of papers (in particular $[2,3,4]$ ) Bezandry and Diagana prove almost periodicity in quadratic mean, which amounts to almost periodicity in probability plus uniform integrability of the square of the norms. We show that Bochneralmost periodicity in distribution and Bochner-almost periodicity in probability are not equivalent, actually neither notion of almost periodicity implies the other. However, under a tightness hypothesis, Bochner-almost periodicity in probability implies Bochner-almost periodicity in distribution.

The paper is organized as follows: The next section is devoted to the case when the state space is metrizable. An extension of the comparison between almost periodicity in distribution and in probability in the completely regular case is given in Section 3 for possible applications in nonmetrizable locally convex topological vector spaces. This extension is done by considering completely regular spaces as inverse limits of metrizable spaces and using the results of section 2. Some complements on criteria of almost periodicity and asymptotic almost periodicity on metric spaces and in completely regular spaces are given in the Appendix (Section 4).

Notations and terminology In all what follows, we are given

- a separable completely regular topological space $\mathbb{E}$ (the state space), e.g. $\mathbb{E}$ is a separable metrizable space,

- an interval $I$, which is $\mathbb{R}$ or $\mathbb{R}^{+}$(the time interval),

- a probability space $(\Omega, \mathcal{F}, \mathrm{P})$,

- a continuous stochastic process $X$ on $\mathbb{E}$ defined on $\Omega \times I$.

We use the following notations:

- We denote by $\mathcal{C}(I ; \mathbb{E})$ the space of continuous functions from $I$ to $\mathbb{E}$.

- The space of trajectories of the process $X$ is the space of continuous functions from $I$ to $\mathbb{E}$, which we endow with the topology of uniform conver- 
gence on compact subsets of $I$ and denote by $\mathcal{C}_{k}=\mathcal{C}_{k}(I ; \mathbb{E})$. The definition of $\mathcal{C}_{k}$ seems to rely on the choice of a uniformity on $\mathbb{E}$ (e.g. of a metric in the metrizable case), but actually the topology of $\mathcal{C}_{k}$ is independent of this choice, see [11, Theorem 8.2.6].

- For any uniform space $F$, we denote $\mathcal{C}_{u}(I ; F)$ the space of continuous functions from $I$ to $F$ endowed with the topology of uniform convergence on $I$.

- For any function $x: I \rightarrow \mathbb{E}$, and for every $t \in I, \widetilde{x}(t)$ is the translation mapping $x(t+):. I \rightarrow \mathbb{E}, s \mapsto x(t+s)$.

- For any topological space $F$, we denote by $\mathcal{P}\left(\mathcal{C}_{k}\right)$ the space of Radon probabilities on $F$ endowed with the topology of narrow convergence, which is the coarsest topology such that the mappings $\mu \mapsto \mu(f)$ are continuous for all bounded continuous functions $f: F \rightarrow \mathbb{R}$.

- We denote by law $(U)$ the law of a random variable $U$. The law of the process $X$ is the law of the $\mathcal{C}_{k}$-valued random variable $X=\widetilde{X}(0)$.

As we concentrate mainly on the study of Bochner-almost periodicity for stochastic processes, by default, in this paper, "almost periodic" means "Bochner-almost periodic".

\section{Metrizable case}

In this section, $\mathbb{E}$ is a Polish space i.e. $\mathbb{E}$ is separable and its topology can be defined by a distance $d_{\mathbb{E}}$ such that $\left(\mathbb{E}, d_{\mathbb{E}}\right)$ is complete.

Before we give the different definitions of almost periodicity for stochastic processes, let us recall the corresponding definitions for deterministic functions.

\subsection{Almost periodicity in the deterministic case}

Let $d_{\mathbb{E}}$ be a distance on $\mathbb{E}$ which is compatible with the topology of $\mathbb{E}$, and let $x: I \rightarrow\left(\mathbb{E} ; d_{\mathbb{E}}\right)$ be a continuous function. We say that $x$ is Bochner-almost periodic if the set $\{\widetilde{x}(t), t \in I\}=\{x(t+),. t \in I\}$ is totally bounded in the space $\mathcal{C}_{u}(I, \mathbb{E})$.

We say that $x$ is Bohr-almost periodic (resp. asymptotically Bohr-almost periodic) if for any $\varepsilon>0$, there exists $l(\varepsilon)>0$ (resp. there exists $l(\varepsilon)>0$ and $T=T(\varepsilon) \geq 0)$ such that any interval of length $l(\varepsilon)$ contains at least an $\varepsilon$-almost period, that is, a number $\tau$ for which

$d_{\mathbb{E}}(x(t+\tau), x(t)) \leq \varepsilon$, for all $t \in I=\mathbb{R}$ (resp. for all $t \geq T$ such that $\left.t+\tau \geq T\right)$. 
In the first case (Bohr-almost periodicity), we say that the set of $\varepsilon$-almost periods of $x$ is relatively dense in $I$. If $\mathbb{E}$ is a vector space, a function $x: \mathbb{R} \rightarrow$ $\mathbb{E}$ is asymptotically Bohr-almost periodic iff it has the form $x=y+z$, where $y$ is Bohr-almost periodic and $z$ is a continuous function with $\lim _{ \pm \infty} z=0$, see e.g. [16].

The following statements are equivalent (see $[6,9]$ and Corollary 4.3):

1. $x$ is Bochner-almost periodic.

2. $x$ satisfies Bochner's double sequence criterion, that is, for every pair of sequences $\left(\alpha_{n}^{\prime}\right)$ and $\left(\beta_{n}^{\prime}\right)$ in $I$, there are subsequences $\left(\alpha_{n}\right) \subset\left(\alpha_{n}^{\prime}\right)$ and $\left(\beta_{n}\right) \subset\left(\beta_{n}^{\prime}\right)$ respectively with same indexes such that, for every $t \in I$, the limits

$$
\lim _{n \rightarrow \infty} \lim _{m \rightarrow \infty} x\left(t+\alpha_{n}+\beta_{m}\right) \text { and } \lim _{n \rightarrow \infty} x\left(t+\alpha_{n}+\beta_{n}\right),
$$

exist and are equal.

3. $x$ is almost periodic in Bohr's sense if $I=\mathbb{R}$, or asymptotically almost periodic in Bohr's sense if $I=\mathbb{R}^{+}$.

Remark 2.1 (i) Bochner's double sequence criterion shows that Bochneralmost periodicity is topological, i.e. it doesn't depend on the choice of $d_{\mathbb{E}}$, provided that $d_{\mathbb{E}}$ is compatible with the topology of $\mathbb{E}$.

(ii) A striking property of Bochner's double sequence criterion is that the limits in (1) exist in any of the three modes of convergences: pointwise, uniform on compact intervals and uniform on $I$ (with respect to $d_{\mathbb{E}}$ ). This criterion has thus the avantage that it allows to establish uniform convergence by checking pointwise convergence.

(iii) Asymptotic Bohr-almost periodicity is strictly weaker than Bohr-almost periodicity, as shows the following example provided by Ruess and Summers [16]:

$$
x(t)=\frac{1}{1+t} \cos [\ln (1+t)], t \in \mathbb{R}^{+} .
$$

\subsection{Almost periodicity and asymptotic almost periodicity in distribution}

Following Tudor's [20] terminology, we say that $X$ is Bochner-almost periodic in distribution, APD for short, (resp. Bohr-almost periodic in distribution, 
resp. asymptotically Bohr-almost periodic in distribution) if the mapping $t \mapsto$ law $(\widetilde{X}(t)), I \rightarrow \mathcal{P}\left(\mathcal{C}_{k}\right)$ is Bochner-almost periodic (resp. Bohr-almost periodic in distribution, resp. asymptotically Bohr-almost periodic in distribution). Other definitions of almost periodicity in distribution are possible, see $[1,13,20]$ : for example we say that $X$ has almost periodic finite dimensional distributions ( $A P F D$ for short) if, for all finite sequences $\left(t_{1}, \ldots, t_{n}\right)$ in $I$, the mapping $t \mapsto$ law $\left(X\left(t_{1}+t\right), \ldots, X\left(t_{n}+t\right)\right)$ is almost periodic. Likewise, if the mapping $t \mapsto$ law $(X(t))$ is almost periodic, we say that $X$ has almost periodic one-dimensional distributions ( $A P O D$ for short).

Bochner-almost periodicity in distribution as defined here is proved for some solutions of stochastic differential equations in $[13,19,1,9]$.

Remark 2.2 If $X$ is almost periodic in distribution, the family $(\widetilde{X}(r))_{r \in I}$ is tight in $\mathcal{C}_{k}$. By [5, Theorem 7.3] or [21, Theorem 4], this implies that, for each interval $[a, b] \subset I$, for every $\varepsilon>0$ and for every $\eta>0$, there exists $\delta>0$ such that

$$
\mathrm{P}\left\{\sup _{\substack{|t-s|<\delta \\ t, s \in[a, b]}} d_{\mathbb{E}}(\tilde{X}(r)(t), \tilde{X}(r)(s))>\eta\right\}<\varepsilon
$$

for all $r \in I$. Equivalently:

(2) $\forall[a, b] \subset I, \forall \varepsilon>0, \forall \eta>0, \exists \delta>0, \forall r \in I$,

$$
\mathrm{P}\left\{\sup _{\substack{|t-s|<\delta \\ t, s \in[a, b]}} d_{\mathbb{E}}(X(r+t), X(r+s))>\eta\right\}<\varepsilon .
$$

Theorem 2.3 If $X$ satisfies (2), the following properties are equivalent:

(a) $X$ is APFD.

(b) $X$ is $A P D$.

Before we give the proof of Theorem 2.3 we recall the following definition: For $f \in C_{b}(\mathbb{E})$, (where $C_{b}(\mathbb{E})$ is the Banach space of continuous and bounded functions defined on $\mathbb{E}$ ),

$$
\begin{gathered}
|f|_{L}=\sup \left\{\frac{|f(x)-f(y)|}{d_{\mathbb{E}}(x, y)} ; x \neq y\right\}, \quad|f|_{\infty}=\sup _{x \in \mathbb{E}}|f(x)|, \\
\text { and }|f|_{B L}=\max \left\{|f|_{\infty},|f|_{L}\right\} .
\end{gathered}
$$


For $\mu, \nu \in \mathcal{P}(E)$, we define

$$
d_{B L}(\mu, \nu)=\sup _{|f|_{B L} \leq 1} \int_{\mathbb{E}} f d(\mu-\nu) .
$$

The metric $d_{B L}$ on $\mathcal{P}(E)$ is complete and compatible with the topology of narrow convergence.

Proof of Theorem 2.3 Clearly $(\mathrm{b}) \Rightarrow(\mathrm{a})$. Assume that $X$ is APFD. Let $\left(\alpha_{n}^{\prime}\right)$ and $\left(\beta_{n}^{\prime}\right)$ be two sequences in $I$ and for $t_{1}, t_{2}, \ldots, t_{k}, t \in I$ define (using notations of $[20])$

$$
\mu_{t}^{t_{1}, \ldots, t_{k}}:=\operatorname{law}\left(X\left(t_{1}+t\right), \ldots, X\left(t_{k}+t\right)\right) .
$$

By a diagonal procedure we can find subsequences $\left(\alpha_{n}\right) \subset\left(\alpha_{n}^{\prime}\right)$ and $\left(\beta_{n}\right) \subset$ $\left(\beta_{n}^{\prime}\right)$ with same indexes such that, for every $k \geq 1$, for all $q_{1}, q_{2}, \ldots, q_{k} \in$ $\mathbb{Q} \cap I$ (where $\mathbb{Q}$ is the set of rational numbers), and for every $t \in I$, the limits

$$
\lim _{n} \mu_{t+\alpha_{n}+\beta_{n}}^{q_{1}, \ldots, q_{k}} \text { and } \lim _{n} \lim _{m} \mu_{t+\alpha_{n}+\beta_{m}}^{q_{1}, \ldots, q_{k}}
$$

exist and are equal. We have, for all $t_{1}, t_{2}, \ldots, t_{k}, t \in I$, and for all $q_{1}, q_{2}, \ldots, q_{k} \in$ $\mathbb{Q} \cap I$

$$
\begin{array}{r}
d_{B L}\left(\mu_{t+\alpha_{n}+\beta_{n}}^{q_{1}, \ldots, q_{k}}, \mu_{t+\alpha_{n}+\beta_{n}}^{t_{1}, \ldots, t_{k}}\right)=\sup _{\|f\|_{B L} \leq 1} \int_{\mathbb{E}^{k}} f d\left(\mu_{t+\alpha_{n}+\beta_{n}}^{q_{1}, \ldots, q_{k}}-\mu_{t+\alpha_{n}+\beta_{n}}^{t_{1}, \ldots, t_{k}}\right) \\
\leq \int_{\Omega} d_{\mathbb{E}^{k}}\left[\left(X\left(q_{1}+t+\alpha_{n}+\beta_{n}\right), \ldots, X\left(q_{k}+t+\alpha_{n}+\beta_{n}\right)\right),\right. \\
\left.\left(X\left(t_{1}+t+\alpha_{n}+\beta_{n}\right), \ldots, X\left(t_{k}+t+\alpha_{n}+\beta_{n}\right)\right)\right] d \mathrm{P} .
\end{array}
$$

Thus, by $(2)$, if $\left(q_{1}, \ldots, q_{k}\right) \rightarrow\left(t_{1}, \ldots, t_{k}\right)$, then

$$
d_{B L}\left(\mu_{t+\alpha_{n}+\beta_{n}}^{q_{1}, \ldots, q_{k}}, \mu_{t+\alpha_{n}+\beta_{n}}^{t_{1}, \ldots, t_{k}}\right) \rightarrow 0
$$

uniformly with respect to $t \in I$ and $n \geq 0$. By a classical result on inversion of limits, we deduce that, for all $k \geq 1$ and $t_{1}, \ldots, t_{k}, t \in I$, the limits

$$
\lim _{n} \mu_{t+\alpha_{n}+\beta_{n}}^{t_{1}, \ldots, t_{k}} \text { and } \lim _{n} \lim _{m} \mu_{t+\alpha_{n}+\beta_{m}}^{t_{1}, \ldots, t_{k}}
$$

exist and are equal. Therefore, to show that the limits

$$
\lim _{n} \operatorname{law}\left(\tilde{X}\left(t+\alpha_{n}+\beta_{n}\right)\right) \text { and } \lim _{n} \lim _{m} \operatorname{law}\left(\tilde{X}\left(t+\alpha_{n}+\beta_{m}\right)\right)
$$


exist and are equal, it is enough to prove that $(\widetilde{X}(t))_{t \in I}$ is tight in $\mathcal{C}_{k}$. Since $X$ is APFD, the family $(X(t))_{t \in I}=(\widetilde{X}(t)(0))_{t \in I}$ is tight, thus by $(2)$ and $[21,5]$ we conclude that $(\widetilde{X}(t))_{t \in I}$ is tight in $\mathcal{C}_{k}$.

Corollary 2.4 Assume that $I=\mathbb{R}^{+}$. If $X$ is an homogeneous Feller process and satisfies (2), the following properties of $X$ are equivalent:

(a) $X$ is $A P O D$.

(b) $X$ is APFD.

(c) $X$ is $A P D$.

Proof The equivalence $(b) \Leftrightarrow(c)$ follows from Theorem 2.3. Clearly (b) $\Rightarrow(a)$. There remains to show that $(a) \Rightarrow(b)$.

Using again Tudor's notations [20], let us set

$$
\mu_{t}:=\text { law }(X(t)) \text { and } \mu_{t}^{t_{1}, \ldots, t_{k}}:=\operatorname{law}\left(\left(X\left(t_{1}+t\right), \ldots, X\left(t_{k}+t\right)\right)\right) .
$$

Suppose that $X$ is APOD, then for every sequence $\left(\alpha_{n}^{\prime}\right) \subset \mathbb{R}^{+}$, there exists a subsequence $\left(\alpha_{n}\right) \subset\left(\alpha_{n}^{\prime}\right)$ such that for each function $g \in C_{b}(\mathbb{E})\left(C_{b}(\mathbb{E})\right.$ is the Banach space of continuous and bounded functions, $g: \mathbb{E} \rightarrow \mathbb{R})$ the limit

$$
\lim _{n} \mu_{t+\alpha_{n}}(g)
$$

exists uniformly with respect to $t \in \mathbb{R}^{+}$. To show that the limit

$$
\lim _{n} \mu_{t+\alpha_{n}}^{t_{1}, \ldots, t_{k}}
$$

exists uniformly with respect to $t \in \mathbb{R}^{+}$, it is enough to show it for $k=2$ and $t_{1}<t_{2}$. Let $f \in C_{b}(\mathbb{E} \times \mathbb{E})$, we have

$$
\mu_{t+\alpha_{n}}^{t_{1}, t_{2}}(f)=\int_{\mathbb{E}} \mu_{0}\left(d x_{0}\right) \int_{\mathbb{E}} p_{t_{1}+t+\alpha_{n}}\left(x_{0}, d x_{1}\right) \int_{\mathbb{E}} p_{t_{2}-t_{1}}\left(x_{1}, d x_{2}\right) f\left(x_{1}, x_{2}\right),
$$

where $p_{t}(x,$.$) is the transition function for a time-homogeneous Markov$ process $X$. Since $X$ is a Feller process, the function

$$
x_{1} \mapsto g\left(x_{1}\right):=\int_{\mathbb{E}} p_{t_{2}-t_{1}}\left(x_{1}, d x_{2}\right) f\left(x_{1}, x_{2}\right)
$$

is in $C_{b}(\mathbb{E})$. Therefore

$$
\mu_{t+\alpha_{n}}^{t_{1}, t_{2}}(f)=\int_{\mathbb{E}} \mu_{0}\left(d x_{0}\right) \int_{\mathbb{E}} p_{t_{1}+t+\alpha_{n}}\left(x_{0}, d x_{1}\right) g\left(x_{1}\right)=\mu_{t+t_{1}+\alpha_{n}}(g)
$$

converges uniformly with respect to $t \in \mathbb{R}^{+}$, hence $X$ is APFD. 


\subsection{Almost periodicity and asymptotic almost periodicity in probability or in $p$-mean}

We say that $X$ is Bochner-almost periodic in probability if $\{\tilde{X}(t), t \in I\}$ is totally bounded with respect to the topology of uniform convergence in probability, which amounts to say that $X$ satisfies Bochner's double sequence criterion for the convergence in probability. Recall from Remark 2.1(ii) that this property depends only on the topology of convergence in probability, not on any metric which is compatible with this topology, thus it is independent of the metric $d_{\mathbb{E}}$ on $\mathbb{E}$.

We say that $X$ is Bohr-almost periodic (resp. asymptotically Bohr-almost periodic) in probability if for any $\varepsilon>0$ and $\eta>0$, there exists $l=l(\varepsilon, \eta)>0$ (resp. there exist $l=l(\varepsilon, \eta)>0$ and $T=T(\varepsilon, \eta)$ ) such that any interval of length $l$ contains at least a number $\tau$ for which

$\mathrm{P}\left\{d_{\mathbb{E}}(X(t+\tau), X(t))>\eta\right\} \leq \varepsilon$, for all $t \in \mathbb{R}$ (resp. for all $\left.t \geq T, t+\tau \geq T\right)$.

Precupanu $[14,15]$ showed that most of the basic properties of Bohralmost periodic functions can be formulated accordingly for $\mathbb{R}$-valued almost periodic in probability processes, particularly, the usual approximation theorem with random trigonometric polynomials is valid for stochastic processes.

Now, let us consider the case when $\mathbb{E}$ is a normed space and $I=\mathbb{R}$. We denote by $\|\cdot\|_{\mathbb{E}}$ the norm of $\mathbb{E}$. Let $p \geq 1$. We say that $X$ is almost periodic in $p$-mean if the mapping $X: \mathbb{R} \rightarrow \mathrm{L}^{p}(\Omega ; \mathbb{E})$ is almost periodic with respect to the metric induced by $\|\cdot\|_{p}=\left(\int\|\cdot\|_{\mathbb{E}}^{p} d P\right)^{\frac{1}{p}}$.

Proposition 2.5 Let $I=\mathbb{R}$. If $X$ is almost periodic in $p$-mean, then it is almost periodic in probability. Conversely, if $X$ is almost periodic in probability and the familly $\left\{\|X(t)\|_{\mathbb{E}}^{p}, t \in \mathbb{R}\right\}$ is uniformly integrable, then $X$ is p-mean almost periodic.

Proof This is a direct consequence of Bochner's double sequence criterion and Vitali Theorem.

Almost periodicity in quadratic mean (using Bohr's definition, but with $I=\mathbb{R}$ ) is proved for some solutions of stochastic differential equations in $[2,3,4]$.

\subsection{Almost sure almost periodicity properties}

We can give at least three different definitions: 
(a) The stochastic process $X$ is almost surely almost periodic if there exists a measurable subset $\Omega_{1} \subset \Omega$ such that $\mathrm{P}\left(\Omega_{1}\right)=1$ and for every $\omega \in \Omega_{1}$, the trajectory $t \mapsto X(t)(\omega)$ is almost periodic.

(b) The stochastic process $X$ satisfies Bochner's almost sure uniform double sequence criterion if, for every pair of sequences $\left(\alpha_{n}^{\prime}\right)$ and $\left(\beta_{n}^{\prime}\right)$ in $I$, there exists a measurable subset $\Omega_{0}$ of $\Omega$ such that $\mathrm{P}\left(\Omega_{0}\right)=1$ and there are subsequences $\left(\alpha_{n}\right) \subset\left(\alpha_{n}^{\prime}\right)$ and $\left(\beta_{n}\right) \subset\left(\beta_{n}^{\prime}\right)$ respectively, with same indexes (independent of $\omega$ ) such that, for every $t \in I$, the limits

$$
\lim _{n \rightarrow \infty} \lim _{m \rightarrow \infty} X\left(t+\alpha_{n}+\beta_{m}\right)(\omega) \text { and } \lim _{n \rightarrow \infty} X\left(t+\alpha_{n}+\beta_{n}\right)(\omega)
$$

exist and are equal for all $\omega \in \Omega_{0}$. (In this case, $\Omega_{0}$ depends on the pair of sequences $\left(\alpha_{n}^{\prime}\right)$ and $\left(\beta_{n}^{\prime}\right)$.)

(c) We say that $X$ is almost equi-almost periodic in Bochner's sense if, for each $\varepsilon>0$, there exists a measurable subset $\Omega_{\varepsilon}$ of $\Omega$ with $\mathrm{P}\left(\Omega_{\varepsilon}\right) \geq$ $1-\varepsilon$ such that the family of trajectories $\{X(\omega)\}_{\omega \in \Omega_{\varepsilon}}$ is equi-almost periodic in Bochner's sense, that is, the family of translation mappings $\left\{\widetilde{X}(t)(\omega) ; t \in I, \omega \in \Omega_{\varepsilon}\right\}$ is totally bounded on $\mathcal{C}_{u}(I, \mathbb{E})$.

Remark 2.6 A stronger notion of almost equi-almost periodicity can be defined as follows:

(d) We say that $X$ is almost surely equi-almost periodic in Bochner's sense if there exists a measurable subset $\Omega_{0} \subset \Omega$ such that $\mathrm{P}\left(\Omega_{0}\right)=1$ and the family of trajectories $\{X(\omega)\}_{\omega \in \Omega_{0}}$ is equi-almost periodic in Bochner's sense.

However this notion seems too strong for stochastic processes: For example, let $U$ be an integer-valued random variable with $P(U=n)>0$ for each integer $n$, and let $X(t)=U$ for every $t \in I$. Then $X$ is almost surely equibounded, and $X$ is also almost periodic in distribution (it is even stationary) and in probability, and it has almost periodic (constant) trajectories. But $X$ is not almost surely equi-almost periodic, since the familly of its trajectories is not almost surely equibounded.

Proposition 2.7 We have (c) $\Rightarrow(\mathrm{b})$ and $(\mathrm{c}) \Rightarrow(\mathrm{a})$.

Remark 2.8 The implications (b) $\Rightarrow(\mathrm{a})$ and $(\mathrm{a}) \Rightarrow(\mathrm{b})$ are false, this will be proved by Counterexamples 2.11 and 2.12. Consequently, the implications $(\mathrm{a}) \Rightarrow(\mathrm{c})$ and $(\mathrm{b}) \Rightarrow(\mathrm{c})$ are false. 
Proof of Proposition 2.7 Assume (c). Let $\left(\alpha_{n}^{\prime}\right)$ and $\left(\beta_{n}^{\prime}\right)$ be two sequences in $I$. For each integer $k \geq 0$, we can apply Bochner's uniform double sequence criterion to the family $\{\widetilde{X}(t)(\omega) ; t \in I\}_{\omega \in \Omega_{2-k}}$ (see Theorem 4.2), that is, we can find subsequences $\left(\alpha_{n}^{k}\right) \subset\left(\alpha_{n}^{\prime}\right)$ and $\left(\beta_{n}^{k}\right) \subset\left(\beta_{n}^{\prime}\right)$ respectively, with same indexes (independent of $\omega$ ) such that, for every $t \in I$, the limits

$$
\lim _{n \rightarrow \infty} \lim _{m \rightarrow \infty} X\left(t+\alpha_{n}^{k}+\beta_{m}^{k}\right)(\omega) \text { and } \lim _{n \rightarrow \infty} X\left(t+\alpha_{n}^{k}+\beta_{n}^{k}\right)(\omega)
$$

exist and are equal for all $\omega \in \Omega_{2^{-k}}$. By induction, we can take $\left(\alpha_{n}^{k+1}\right) \subset$ $\left(\alpha_{n}^{k}\right)$ and $\left(\beta_{n}^{k+1}\right) \subset\left(\beta_{n}^{k}\right)$. Set $\alpha_{n}=\alpha_{n}^{n}$ and $\beta_{n}=\beta_{n}^{n}$ and $\Omega_{0}=\liminf \Omega_{2^{-k}}$. Then $\mathrm{P}\left(\Omega_{0}\right)=1$ and the limits (3) exist and are equal for all $\omega \in \Omega_{0}$. Thus (b) holds true. Furthermore, $\Omega_{0}$ does not depend on $\left(\alpha_{n}^{\prime}\right)$ and $\left(\beta_{n}^{\prime}\right)$. Thus, for every $\omega \in \Omega_{0}, X(\omega)$ is almost periodic, which proves (a).

\subsection{Comparison between almost periodicities}

\section{Almost periodicity in probability vs almost sure properties}

Lemma 2.9 Let $\left(X_{n}(t)\right)$ be a sequence of processes which converges in probability uniformly with respect to $t \in I$. There exists a subsequence $\left(X_{n_{k}}(t)\right) \subset$ $\left(X_{n}(t)\right)$ which converges almost surely for each $t \in I$.

Proof Assume that $\left(X_{n}(t)\right)$ converges in probability to a process $X(t)$ uniformly with respect to $t \in I$, let us denote $Y_{n}(t)=d\left(X_{n}(t), X(t)\right)$. Then for each $\varepsilon>0$ and $\eta>0$, there exists $N=N(\varepsilon, \eta)$ such that for all $t \in I$ and $n \geq N$, we have

$$
\mathrm{P}\left(Y_{n}(t)>\varepsilon\right)<\eta .
$$

Let $\left(\eta_{k}\right)$ be a real positive sequence such that

$$
\sum_{k \geq n} \eta_{k} \text { converges to zero as } n \rightarrow \infty \text {. }
$$

To each $\varepsilon>0$ and $\eta_{k}>0$, there corresponds $N_{k}=N\left(\varepsilon, \eta_{k}\right)$ such that, for all $t \in I, \mathrm{P}\left(Y_{N_{k}}(t)>\varepsilon\right)<\eta_{k}$. Let us show that $\left(Y_{N_{k}}(t)\right)$ converges almost surely for every $t \in I$. We have

$$
\mathrm{P}\left(\sup _{k \geq n} Y_{N_{k}}(t)>\varepsilon\right)=\mathrm{P}\left(\bigcup_{k \geq n}\left\{Y_{N_{k}}(t)>\varepsilon\right\}\right) \leq \sum_{k \geq n} \eta_{k}
$$

and the right hand term converges to zero when $n \rightarrow \infty$. Thus $\left(Y_{N_{k}}(t)\right)$ converges almost surely to zero for every $t \in I$. Consequently $\left(X_{N_{k}}(t)\right)$ converges almost surely to $X(t)$ for every $t \in I$. 
Theorem 2.10 The following properties of $X$ are equivalent:

(1) X satisfies Bochner's almost sure uniform double sequence criterion.

(2) $X$ is almost periodic in probability.

Proof Obviously $(1) \Rightarrow(2)$.

Assume that $X$ is almost periodic in probability. Let $\left(\alpha_{n}^{\prime}\right)$ and $\left(\beta_{n}^{\prime}\right)$ be two sequences in $I$. There exist subsequences $\left(\alpha_{n}\right) \subset\left(\alpha_{n}^{\prime}\right)$ and $\left(\beta_{n}\right) \subset\left(\beta_{n}^{\prime}\right)$ with same indexes such that for each $t \in I$, the limits in probability

$$
\mathrm{P}-\lim _{n} X\left(\alpha_{n}+\beta_{n}+t\right) \text { and } \mathrm{P}-\lim _{n} \lim _{m} X\left(\alpha_{n}+\beta_{m}+t\right)
$$

exist and are equal. By Remark 2.1(ii) these limits exist uniformly with respect to $t \in I$. The previous Lemma implies that there exist further subsequences (still denoted $\left(\alpha_{n}\right)$ and $\left(\beta_{n}\right)$ ) with same indexes such that, for each $t \in I$, the limits

$$
\lim _{n} X\left(\alpha_{n}+\beta_{n}+t\right) \text { and } \lim _{n} \lim _{m} X\left(\alpha_{n}+\beta_{m}+t\right)
$$

exist and are equal almost surely.

We now give counterexamples which show that $X$ can be almost periodic in probability without any almost periodic trajectory, or almost surely almost periodic without being almost periodic in probability. By Theorem 2.10, these counterexamples show that the implications (a) $\Rightarrow$ (c) and (b) $\Rightarrow$ (c) in Proposition 2.7 are false. Consequently, the converse implications to (c) $\Rightarrow$ (a) and (c) $\Rightarrow$ (b) in Proposition 2.7 are also false.

Counterexample 2.11 (almost periodicity in probability without almost periodic trajectories) Let $\left(\delta_{n}\right)$ be the sequence of random variables on the probability space $([0,1], d t)$ ( $d t$ is the Lebesgue measure) defined by $\delta_{n}=1_{\left[\frac{n}{2^{k}}, \frac{n+1}{\left.2^{k}\right]}\right.}$ for $2^{k-1} \leq n<2^{k}, k \in \mathbb{N}$. For each $n \geq 1$, let $f_{n}:[0,1] \rightarrow \mathbb{R}$ be defined by

$$
f_{n}(t)= \begin{cases}n t & \text { if } t \in\left[0, \frac{1}{2}\right] \\ n(1-t) & \text { if } t \in\left[\frac{1}{2}, 1\right]\end{cases}
$$

and let $\left(X_{t}\right)_{t \geq 0}$ be defined by

$$
X_{t}(\omega)=\left\{\begin{array}{lll}
f_{[t]}(t-[t]) & \text { if } & \delta_{[t]}(\omega)=1 \\
0 & \text { if } & \delta_{[t]}(\omega)=0
\end{array}\right.
$$


where $[t]$ is the integer part of $t$. The trajectories of $X$ are continuous on $\mathbb{R}^{+}$, but almost none is uniformly continuous, nor bounded, since $\mathrm{P}\left(\lim \sup \delta_{n}=\right.$ $1)=1$. We have, for every $\varepsilon>0$,

$$
\lim _{t \rightarrow \infty} \mathrm{P}\left(X_{t}>\varepsilon\right) \leq \lim _{t \rightarrow \infty} \mathrm{P}\left(\delta_{[t]}=1\right)=0
$$

which means that $X$ is asymptotically Bohr-almost periodic in probability, thus it is Bochner-almost periodic in probability.

A variant of this example, with uniformly bounded trajectories, is obtained by replacing $X$ by $\min (X, 1)$.

Note that, for all integers $n, p \geq 0$ and for every $\eta>0$ and every $\delta>0$, we have

$$
\begin{array}{r}
\mathrm{P}\left\{\sup _{\substack{|t-s|<\delta \\
t, s \in[n, n+p]}} d_{\mathbb{E}}(X(t), X(s))>\eta\right\} \leq \mathrm{P}\left\{\exists k \in\{n, \ldots, n+p\} ; \delta_{k}=1\right\} \\
\leq 2^{-n+1} \rightarrow 0 \text { when } n \rightarrow \infty .
\end{array}
$$

As the trajectories of $X$ are $n$-Lipschitz on $[0, n]$, this implies that $X$ satisfies Condition (2). We shall see later that $X$ is almost periodic in distribution, thus Counterexample 2.11 also provides an example of an almost periodic in distribution process without any almost periodic trajectory.

Counterexample 2.12 (almost sure almost periodicity does not imply almost periodicity in probability) Let $X$ be defined by $X(t)(\omega)=$ $e^{i \frac{t}{\omega}}$, for every $\left.\left.\omega \in\right] 0,1\right]$ and $t \in I$. The process $X$ is almost surely almost periodic, but it is not almost periodic in probability (thus it is not almost equi-almost periodic). Indeed, since $|X| \leq 1$ then to prove that $X$ is not almost periodic in probability, il suffices to show that $X$ is not almost periodic in quadratic mean. Let $\tau$ be an $\varepsilon$-almost period i.e. $E\left|X_{t+\tau}-X_{t}\right|^{2} \leq \varepsilon$, for all $t \in I$. Let $A(\tau):=E\left|X_{t+\tau}-X_{t}\right|^{2}$,

$$
\begin{aligned}
A(\tau) & =\int_{0}^{1}\left|e^{i \frac{t+\tau}{\omega}}-e^{i \frac{t}{\omega}}\right|^{2} d \omega=\int_{0}^{1}\left|e^{i \frac{\tau}{\omega}}-1\right|^{2} d \omega=4 \int_{0}^{1}\left|\frac{e^{i \frac{\tau}{2 \omega}}-e^{-i \frac{\tau}{2 \omega}}}{2 i}\right|^{2} d \omega \\
& =2 \int_{0}^{1} \sin ^{2}\left(\frac{\tau}{w}\right) d \omega=2|\tau| \int_{|\tau|}^{\infty} \frac{1-\cos (2 u)}{u^{2}} d u \\
& =2|\tau| \sum_{k=0}^{\infty} \int_{|\tau|+k \pi}^{|\tau|+(k+1) \pi} \frac{1-\cos (2 u)}{u^{2}} d u \geqslant 2|\tau| \int_{|\tau+\pi|}^{\infty} \frac{1}{u^{2}} d u=2 \frac{|\tau|}{|\tau|+\pi} .
\end{aligned}
$$

Thus if $|\tau| \geqslant \frac{\varepsilon \pi}{2-\varepsilon}$, we get $A(\tau)>\varepsilon$, which shows that the set of $\varepsilon$-almost periods is not relatively dense in $I$, thus $X$ is not almost periodic in quadratic mean. 


\section{Almost periodicity in distribution vs other almost periodicity prop- erties}

Remark 2.13 In all preceding definitions of almost periodicity, it is only in the definition of almost periodicity in distribution that the choice of a topology on the space $\mathcal{C}(I, \mathbb{E})$ of trajectories plays a role.

Theorem 2.14 (Almost periodicity in distribution vs almost periodicity in probability) Consider the following properties of $X$ :

(a) $X$ is $A P D$.

(b) $X$ is APFD.

(c) $X$ is almost periodic in probability.

We have (c) $\Rightarrow(\mathrm{b})$. If furthermore $X$ satisfies (2), then (c) $\Rightarrow$ (a).

Remark 2.15 The implications (c) $\Rightarrow$ (a) and (a) $\Rightarrow$ (c) are false, see Counterexamples 2.16 and 2.17.

Proof of Theorem 2.14 Assume (c) and let $\left(\alpha_{n}^{\prime}\right)$ and $\left(\beta_{n}^{\prime}\right)$ be two sequences in $I$. There exist subsequences $\left(\alpha_{n}\right) \subset\left(\alpha_{n}^{\prime}\right)$ and $\left(\beta_{n}\right) \subset\left(\beta_{n}^{\prime}\right)$ with same indexes such that for each $t, s \in I$, the limits in probability

$$
\mathrm{P}-\lim _{n} X\left(\alpha_{n}+\beta_{n}+t+s\right) \text { and } \mathrm{P}-\lim _{n} \lim _{m} X\left(\alpha_{n}+\beta_{m}+t+s\right)
$$

exist and are equal. Hence, for every $\left(t_{1}, t_{2}, \ldots, t_{k}\right) \in I^{k}$, and every $s \in I$

$\mathrm{P}-\lim _{n}\left(X\left(\alpha_{n}+\beta_{n}+t_{1}+s\right), X\left(\alpha_{n}+\beta_{n}+t_{2}+s\right), \ldots, X\left(\alpha_{n}+\beta_{n}+t_{k}+s\right)\right)$

and

$\mathrm{P}-\lim _{n} \lim _{m}\left(X\left(\alpha_{n}+\beta_{m}+t_{1}+s\right), X\left(\alpha_{n}+\beta_{m}+t_{2}+s\right), \ldots, X\left(\alpha_{n}+\beta_{m}+t_{k}+s\right)\right)$

exist and are equal, thus

$$
\lim _{n} \operatorname{law}\left(X\left(\alpha_{n}+\beta_{n}+t_{1}+s\right), \ldots, X\left(\alpha_{n}+\beta_{n}+t_{k}+s\right)\right)
$$

and

$$
\lim _{n} \lim _{m} \operatorname{law}\left(\left(X\left(\alpha_{n}+\beta_{m}+t_{1}+s\right), \ldots, X\left(\alpha_{n}+\beta_{m}+t_{k}+s\right)\right)\right.
$$

exist and are equal, that is, $X$ is APFD, and by Theorem 2.3 we deduce (a). 
Counterexample 2.16 (almost periodicity in distribution does not imply almost periodicity in probability) Let $X$ be a strictly stationary (thus APD) continuous process such that $\left(X_{n}\right)_{n \in \mathbb{Z}}$ is independent and $X_{0}$ is nonconstant.

Assume that $X$ is almost periodic in probability. Then we can extract from $\left(X_{n}\right)$ a subsequence $\left(X_{n_{k}}\right)$ which converges in probability to some random variable $U$. By stationarity, we have law $(U)=$ law $\left(X_{0}\right)$. Extracting if necessary a further subsequence, we can assume that $\left(X_{n_{k}}\right)$ converges a.e. to $U$. By Kolmogorov's zero-one law, $\mathrm{U}$ is constant a.e., a contradiction. Thus $X$ is not almost periodic in probability.

To construct such a process $X$, we can use results of [10]. Let $\sigma$ be an atomless probability measure on $[0, \pi[$, and let $\psi(u)=\inf \{x ; \sigma([0, x]) \geq u\}$. Let $\gamma$ be the symmetric measure on $[-\pi, \pi]$ defined by

$$
\gamma(A)=\frac{1}{2}(\sigma(A \cap[0, \pi])+\sigma(-A \cap[0, \pi]))
$$

for every Borel subset of $[-\pi, \pi]$. Let $\left(B_{u}\right)_{0 \leq u \leq 1}$ be a standard Brownian motion on the complex plane, and define a complex Gaussian centered process $\left(\xi_{t}\right)_{t \in \mathbb{R}}$ by

$$
\xi_{t}=\int_{0}^{1} e^{i t \psi(u)} d B_{u}
$$

By the construction in [10], we have $\xi_{t}=B_{1} \circ T^{t}$, where $\left(T^{t}\right)$ is a stationary flow on the probability space on which $B$ is defined, thus $\xi$ is strictly stationary. Furthermore, by [10, Proposition 3.1], almost all trajectories of $\xi$ are $C^{\infty}$. Let $X$ be the real part of $\xi$. The process $X$ is Gaussian stationary continuous and centered, and, by [10, Proposition 2.2], we have

$$
\mathrm{E}\left(X_{n} X_{m}\right)=\int_{-\pi}^{\pi} e^{i t(n-m)} d \gamma(t)
$$

for all $n, m \in \mathbb{Z}$. In particular, if $\sigma$ is the normalized Lebesgue measure, $\left(X_{n}\right)_{n \in \mathbb{Z}}$ is independent.

Counterexample 2.17 (almost periodicity in probability does not imply almost periodicity in distribution) This example is constructed in the spirit of Counterexample 2.11, but the process constructed in Counterexample 2.11 is almost periodic in probability and satisfies Condition (2), thus by Theorem 2.14 it is almost periodic in distribution. The process constructed here is almost periodic in probability but does not satisfy Condition (2). 
Let $\Omega=[0,1[$ endowed with the Lebesgue measure. For every integer $n \geq 0$ and every $\omega \in\left[0,1\left[\right.\right.$, let $\omega_{n}^{\prime}=\min (\omega+1 / n, 1)$. Define a function $f_{n}$ on $[0,1[\times[0,1[$ by

$$
f_{n}(\omega, t)=\left\{\begin{array}{lll}
0 & \text { if } & t \leq \omega \text { or } t \geq \omega_{n}^{\prime} \\
2 \frac{t-\omega}{\omega_{n}^{\prime}-\omega} & \text { if } & \omega \leq t \leq \frac{\omega+\omega_{n}^{\prime}}{2} \\
2 \frac{\omega_{n}^{\prime}-t}{\omega_{n}^{\prime}-\omega} & \text { if } & \frac{\omega+\omega_{n}^{\prime}}{2} \leq t \leq \omega_{n}^{\prime}
\end{array}\right.
$$

For $t \geq 0$ and $\omega \in[0,1[$, set

$$
X_{t}(\omega)=f_{[t]}(\omega, t-[t]),
$$

where $[t]$ denotes the integer part of $t$. Then $X$ has continuous trajectories, and we have, for every $\varepsilon>0$,

$$
\lim _{t \rightarrow \infty} \mathrm{P}\left(X_{t}>\varepsilon\right) \leq \lim _{t \rightarrow \infty} \frac{1}{[t]}=0
$$

which means that $X$ is asymptotically Bohr-almost periodic in probability thus it is Bochner-almost periodic in probability. On the other hand, for each $n \geq 0$ and for each $\omega \in\left[0,1\left[, f_{n}(\omega,\right.\right.$.$) takes the value 1$ at the point $\tau_{n}(\omega)=\left(\omega+\omega_{n}^{\prime}\right) / 2$ and the value 0 outside the interval $\left[\omega, \omega_{n}^{\prime}\right]$. Thus, for each integer $n \geq 0$ and for each $\omega \in \Omega$, there exist $t=n+\tau_{n}(\omega) \in[n, n+1[$ such that $X_{t}(\omega)=1$ and $s \in\left[n, n+1\left[\operatorname{such}\right.\right.$ that $X_{s}(\omega)=0$ and $|t-s| \leq 1 / n$. For every $\delta>0$, and for $n \geq 1 / \delta$, we thus have

$$
\mathrm{P}\left\{\sup _{\substack{|t-s|<\delta \\ t, s \in[n, n+1]}} d_{\mathbb{E}}(X(t), X(s))>1 / 2\right\}=1,
$$

which contradicts Condition (2). Thus $X$ is not almost periodic in distribution.

Theorem 2.18 If $X$ is almost equi-almost periodic, then $X$ is almost periodic in probability and in distribution.

Proof By Proposition 2.7 and Theorem 2.10, $X$ satisfies Bochner's almost sure uniform double sequence criterion which is equivalent to almost periodicity in probability.

Now, by Theorem 2.14, to prove that $X$ is APD, we only need to show that $X$ satisfies (2). Since $X$ is almost equi-almost periodic, almost all its 
trajectories are uniformly continuous, thus we can find for each $\omega \in \Omega$ and for each $\eta>0$, a number $\delta(\omega)>0$, such that, for all $t, s \in I$,

$$
\sup _{|t-s|<\delta(\omega)} d_{\mathbb{E}}(X(t)(\omega), X(s)(\omega))<\eta .
$$

Furthermore, by restricting to the rational numbers the variables $t$ and $s$ in (4), we can assume that the mapping $\omega \mapsto \delta(\omega)$ is measurable. For each $\varepsilon>0$, there exists $\delta_{\varepsilon}>0$ such that

$$
\mathrm{P}\left\{\delta>\delta_{\varepsilon}\right\}>1-\varepsilon .
$$

Indeed, suppose that (5) is false, then there exists $\varepsilon_{0}>0$ such that for every $\delta^{*}>0$, we have $\mathrm{P}\left\{\delta \leq \delta^{*}\right\}>\varepsilon_{0}$. In particular, for each $m \in \mathbb{N}$ $\mathrm{P}\left\{\delta \leq \frac{1}{m}\right\}>\varepsilon_{0}$. Let $A_{m}=\left\{\delta \leq \frac{1}{m}\right\}$, we have

$$
\mathrm{P}\{\delta=0\}=\inf _{m} \mathrm{P}\left\{\delta \leq \frac{1}{m}\right\}>\varepsilon_{0}>0,
$$

which contradicts $\delta(\omega)>0$ for every $\omega \in \Omega$. Thus, from (4) and (5), we deduce that there exists $\delta_{\varepsilon}$ such that

$$
\mathrm{P}\left\{\sup _{|t-s|<\delta_{\varepsilon}} d_{\mathbb{E}}(X(t), X(s))>\eta\right\} \leq \varepsilon .
$$

Therefore, for every interval $[a, b] \subset I$ and every $r \in I$, we have

$$
\mathrm{P}\left\{\sup _{\substack{|t-s|<\delta_{\varepsilon} \\ t, s \in[a, b]}} d_{\mathbb{E}}(\tilde{X}(r)(t), \widetilde{X}(r)(s))>\eta\right\} \leq \varepsilon
$$

i.e. $X$ satisfies (2).

\section{$3 \quad$ Extension to completely regular state spaces}

Topological preliminaries We now assume that $\mathbb{E}$ is a separable completely regular space. Recall that a Hausdorff topological space is completely regular iff it is uniformizable, that is, iff its topology can be defined by a family of semi-metrics. Such a family defines a uniformity. The notions of uniform continuity, Cauchy sequences, Cauchy nets and totally bounded sets are defined relatively to a given uniformity. For more details see e.g. [11].

Let $\mathfrak{U}$ be a uniformity (a set of entourages) on $\mathbb{E}$ which is compatible with the topology of $\mathbb{E}$, and let $\mathfrak{D}$ be an upwards filtering set of semimetrics 
on $\mathbb{E}$ which induces this uniformity. For every $d \in \mathfrak{D}$, we denote by $\breve{\mathbb{E}}_{d}$ the quotient metric space $(\mathbb{E} / d, \breve{d})$, and by $\breve{\mathcal{C}}_{k}^{d}$ the space $\mathcal{C}_{k}\left(I ; \breve{\mathbb{E}}_{d}\right)$. We denote by $p_{d}$ the projection mapping:

$$
p_{d}:\left\{\begin{array}{lll}
\mathbb{E} & \rightarrow & \breve{\mathbb{E}}_{d} \\
x & \mapsto & \breve{x}_{d}
\end{array}\right.
$$

which extends to $\mathcal{C}_{k}$ :

$$
p_{d}: \begin{cases}\mathcal{C}_{k} & \rightarrow \breve{\mathcal{C}}_{k}^{d} \\ x & \mapsto p_{d} \circ x=: \breve{x}_{d}(.)\end{cases}
$$

These mappings are continuous and the uniform space $\mathbb{E}$ (resp. $\mathcal{C}_{k}$ ) is the limit of the inverse system $\left\{\breve{\mathbb{E}}_{d}\right\}_{d \in \mathfrak{D}}\left(\right.$ resp. $\left.\left\{\breve{\mathcal{C}}_{k}^{d}\right\}_{d \in \mathfrak{D}}\right)$.

Almost periodicity for deterministic functions A function $x$ in $\mathcal{C}(I ; \mathbb{E})$ is said to be Bochner-almost periodic, if the set $\{x(t+),. t \in I\}$ of its translation mappings is totally bounded on $\mathcal{C}_{u}(I ; \mathbb{E})$.

A function $x$ in $\mathcal{C}(\mathbb{R} ; \mathbb{E})$ is said to be Bohr-almost periodic if for each entourage $V$ in $\mathfrak{U}$, there exists a real number $l=l(V)>0$ such that every interval $[a, a+l]$ contains at least one point $\tau$ satisfying

$$
(x(t+\tau), x(t)) \in V \text {, for all } t \in \mathbb{R} .
$$

We say that a function $x \in \mathcal{C}\left(\mathbb{R}^{+} ; \mathbb{E}\right)$ is asymptotically Bohr-almost periodic if, for each entourage $V \in \mathfrak{U}$, there exist a real number $l=l(V)>0$ and $T=T(V)>0$ with the property that any interval $[a, a+l] \subset \mathbb{R}^{+}$contains a number $\tau$ such that

$$
(x(t+\tau), x(t)) \in V, \text { for all } t \geq T \text { such that } t+\tau \geq T .
$$

The relationships between these notions of almost periodicity are explained in Lemma 4.4 in the Appendix.

Almost periodicity in distribution vs in probability for stochastic processes Let $\mathcal{P}(\mathbb{E})$ (resp. $\mathcal{P}\left(\mathcal{C}_{k}\right)$ ) be the space of Radon probabilities on $\mathbb{E}$ (resp. $\mathcal{C}_{k}$ ) endowed with the initial topology of narrow convergence, that is the coarsest topology such that the mappings $\mu \rightarrow \mu(f)$ are continuous for all measurable bounded function $f: \mathbb{E} \rightarrow \mathbb{R}$ (resp. $f: \mathcal{C}_{k} \rightarrow \mathbb{R}$ ). For more details on this topology, see [18]. 
For each projection mapping $p_{d}$, we can define a mapping from $\mathcal{P}\left(\mathcal{C}_{k}\right)$ to $\mathcal{P}\left(\breve{\mathcal{C}}_{k}^{d}\right)$ (or from $\mathcal{P}(\mathbb{E})$ to $\mathcal{P}\left(\breve{\mathbb{E}}_{d}\right)$ ) by

$$
\mu \rightarrow\left(p_{d}\right)_{\sharp}(\mu)=\breve{\mu}^{d}
$$

where $\left(p_{d}\right)_{\sharp}(\mu)$ denotes the image of $\mu$ by $p_{d}$ that is, $\breve{\mu}^{d}(f)=\mu\left(f \circ p_{d}\right)$ for every measurable bounded function $f: \breve{\mathcal{C}}_{k}^{d} \rightarrow \mathbb{R}$.

We say that the process $X$ is almost periodic in distribution (resp. asymptotically almost periodic in distribution) in Bohr's or Bochner sense if the mapping

$$
\left\{\begin{aligned}
I & \rightarrow \mathcal{P}\left(\mathcal{C}_{k}\right) \\
t & \mapsto \operatorname{law}(\widetilde{X}(t))
\end{aligned}\right.
$$

is almost periodic (resp. asymptotically almost periodic) in Bohr's or Bochner sense in $\mathcal{P}\left(\mathcal{C}_{k}\right)$.

We say that $X$ is Bochner-almost periodic in probability if the $\operatorname{set}\{\widetilde{X}(t), t \in I\}$ of translation mappings is totally bounded in probabilily.

We say that $X$ is Bohr-almost periodic (resp. asymptotically Bohr-almost periodic) in probability if for any entourage $V$ and $\eta>0$, there exists $l=$ $l(V, \eta)>0$ (resp. there exist $l=l(V, \eta)>0$ and $T=T(V, \eta))$ such that any interval of length $l$ contains at least a number $\tau$ for which

$P\{(X(t+\tau), X(t)) \notin V\} \leq \eta$, for all $t \in \mathbb{R}$ (resp. for all $t \geq T, t+\tau \geq T)$.

In order to establish a result similar to Theorem 2.14, we need to characterize total boundedness in inverse limits of uniform spaces.

Lemma 3.1 Let $\left\{\mathbb{F}_{\rho}\right\}_{\rho \in \Sigma}$ be an inverse system of uniform spaces where $\Sigma$ is directed set, and let

$$
\mathbb{F}=\lim _{\longleftarrow}\left\{\mathbb{F}_{\rho}\right\}
$$

the projective limit satisfying $\mathbb{F}_{\rho}=p_{\rho}(\mathbb{F})$, for every projection mapping $p_{\rho}$ from $\mathbb{F}$ to $\mathbb{F}_{\rho}$. Then, $\mathbb{F}$ is totally bounded if and only if for each $\rho \in \Sigma, \mathbb{F}_{\rho}$ is totally bounded.

Proof It is straightforward that, if $\mathbb{F}$ is totally bounded, $\mathbb{F}_{\rho}$ is also totally bounded for each $\rho \in \Sigma$.

Conversely, assume that for each $\rho \in \Sigma, \mathbb{F}_{\rho}$ is totally bounded. Let $\widehat{\mathbb{F}_{\rho}}$ be the completion of $\mathbb{F}_{\rho}$, then $\widehat{\mathbb{F}_{\rho}}$ is a compact space and $\left\{\widehat{\mathbb{F}_{\rho}}, \rho \in \Sigma\right\}$ is 
an inverse system (see [18, second proof of Prokhorov theorem, page 78]). Hence the projective limit

$$
\widehat{\mathbb{F}}:=\lim _{\longleftarrow} \widehat{\mathbb{F}} \rho
$$

is a compact space (see [7, page I.64, Proposition 8]). On the other hand, using [11, Proposition 2.5.6. page 100] one can see that $\overline{\mathbb{F}_{\rho}}, \rho \in \Sigma$, is an inverse system satisfying

$$
\lim _{\longleftarrow} \overline{\mathbb{F}_{\rho}}=\overline{\mathbb{F}},
$$

where $\overline{\mathbb{F}} \rho$ is the closure of $\mathbb{F}_{\rho}$ in $\widehat{\mathbb{F}} \rho$ and $\overline{\mathbb{F}}$ is the closer of $\mathbb{F}$ in $\widehat{\mathbb{F}}$, which yields $\overline{\mathbb{F}}=\widehat{\mathbb{F}}$, thus $\mathbb{F}$ is totally bounded.

Now we can state and prove a version of Theorem 2.14 in the case of a process with values in a completely regular space $\mathbb{E}$.

Theorem 3.2 Consider the following properties of $X$ :

(a) $X$ is almost periodic in distribution.

(b) $X$ is almost periodic in probability.

(c) for each interval $[a, b] \subset I$, for every $\varepsilon>0$ and for every $\eta>0$, there exists $\delta>0$ such that for every $d \in \mathfrak{D}$

$$
\mathrm{P}\left\{\sup _{\substack{|t-s|<\delta \\ t, s \in[a, b]}} d(X(r+t), X(r+s))>\eta\right\}<\varepsilon
$$

for all $r \in I$.

Then $((\mathrm{c})$ and $(\mathrm{b})) \Rightarrow(\mathrm{a})$.

Proof Assume that $X$ is almost periodic in probability and satisfies Property (c). Then for each $d \in \mathfrak{D}$ the process $X^{d}=p_{d} \circ X$ satisfies (2) (see Section 2) and by Lemma $4.4 X^{d}$ is almost periodic in probability in $\mathbb{E}_{d}$ for each $d \in \mathfrak{D}$, hence $X^{d}$ is almost periodic in probability in the completion $\widehat{\mathbb{E}_{d}}$ (of $\breve{\mathbb{E}}_{d}$ ) for each $d \in \mathfrak{D}$. In view of Theorem 2.14 we get that $X^{d}$ is almost periodic in distribution in $\widehat{\mathbb{E}_{d}}$, i.e. the set $\left\{\widetilde{\mu}_{t+.}^{d} ; t \in I\right\}$ of translation mappings is totally bounded on the space of functions on $I$ with values in the space $\mathcal{P}\left(\breve{\mathcal{C}}_{k}^{d}\right)$. By Prokhorov Theorem on inverse systems of Radon measures (see [18, Theorem 21, page 74]), it is easy to see that the set $\left\{\widetilde{\mu}_{t+.}^{d} ; t \in I\right\}$ is an exact inverse system and that its projective limit is the set $\left\{\tilde{\mu}_{t+.} ; t \in I\right\}$ of translation mappings. Now, applying Lemma 3.1 to this set, we obtain that it is totally bounded, that is, $X$ is almost periodic in distribution. 


\section{Appendix : Almost periodicity and asymptotic almost periodicity in completely regular spaces}

First we consider the case when $\mathbb{E}$ is a metric space.

Bochner's criteria of almost periodicity for functions with values in a metric space Let $(\mathbb{E}, d)$ be a metric space (not necessarily complete). A subset $\mathcal{A} \subset I$ is said to be relatively dense if there exists a real number $l>0$ such that for each number $a \in I,[a, a+l] \cap \mathcal{A} \neq \emptyset$. Any such number $l$ is called an inclusion length of $\mathcal{A}$.

A subset $\mathcal{H}$ of $\mathcal{C}(I ; \mathbb{E})$ is said to be equi-almost periodic (respectively equi-asymptotically almost periodic) in Bohr's sense if, for every $\varepsilon>0$, there exists a relatively dense set $\mathcal{A}=\mathcal{A}(\mathcal{H}, \varepsilon)$ in $I$ (resp. there exist a relatively dense set $\mathcal{A}=\mathcal{A}(\mathcal{H}, \varepsilon)$ in $I$ and a number $r \geq 0$ ) such that, for every $\tau \in \mathcal{A}$ and $h \in \mathcal{H}$,

$$
d(h(t+\tau), h(t))<\varepsilon \text { for all } t \in \mathbb{R} \text { (resp. for all } t \geq r, t+\tau \geq r) .
$$

A subset $\mathcal{H}$ of $\mathcal{C}(I ; \mathbb{E})$ is said to be equinormal if, from every sequence $\left(\alpha_{n}^{\prime}\right)$ in $I$, one may extract a subsequence $\left(\alpha_{n}\right)$ such that the sequence $\left(h\left(t+\alpha_{n}\right)\right)$ converges uniformly with respect to $t \in I$ and $h \in \mathcal{H}$.

The following results are adaptations of those obtained by Ruess and Summers [17] in the case of locally convex spaces. We only consider the case $I=\mathbb{R}^{+}$, the almost periodic case follows exactly in the same way with obvious minor changes.

Lemma 4.1 Let $\mathcal{H}$ be a subset of $\mathcal{C}\left(\mathbb{R}^{+} ; \mathbb{E}\right)$. Suppose that $\mathcal{H}$ is a totally bounded in $\mathcal{C}_{k}\left(\mathbb{R}^{+} ; \mathbb{E}\right)$ and equi-asymptotically almost periodic. Then, $\mathcal{H}$ is uniformly equicontinuous on $\mathbb{R}^{+}$and $\mathcal{H}\left(\mathbb{R}^{+}\right)$is totally bounded in $\mathbb{E}$.

Proof Let $\varepsilon>0$, since $\mathcal{H}$ is equi-asymptotically almost periodic in Bohr's sense, there exist a number $r=r(\varepsilon)>0$ and a relatively dense set $\mathcal{A}=$ $\mathcal{A}(\mathcal{H}, \varepsilon)$ in $[r,+\infty[$ such that, for each $\tau \in \mathcal{A}$ and for every $h \in \mathcal{H}$,

$$
d(h(t+\tau), h(t))<\varepsilon \text { for all } t \geq r \text { such that } t+\tau \geq r .
$$

Let $N=\max (r, l)$, where $l$ is an inclusion length of $\mathcal{A}$. Choose $\tau_{k} \in[k N,(k+$ 1) $N], k=1,2, \ldots$. Let us denote by $\mathcal{H} /[0,5 N]$ the restriction of all functions of $\mathcal{H}$ to the interval $[0,5 N]$. Then $\mathcal{H} /[0,5 N]$ is totally bounded in $\mathcal{C}([0,5 N] ; \mathbb{E})$. Hence, by Ascoli Theorem, we get that $\mathcal{H} /[0,5 N]$ is uniformly equicontinuous. Let $\delta \in\left[0, \frac{N}{2}\right]$ such that $d\left(h\left(t_{1}\right), h\left(t_{2}\right)\right)<\frac{\varepsilon}{3}$ whenever $h \in \mathcal{H}$ and $t_{1}, t_{2} \in$ 
$[0,5 N]$ with $\left|t_{1}-t_{2}\right|<\delta$. Now, suppose that $t_{1}, t_{2}>4 N$, with $\left|t_{1}-t_{2}\right|<\delta$. Taking $k \in \mathbb{N}$ such that $t_{1}, t_{2} \in[k N,(k+2) N]$, let $s_{i}=t_{i}-\tau_{k-2}, i=1,2$. Since $s_{1}, s_{2} \in[N, 4 N]$ and $\left|s_{1}-s_{2}\right|<\delta$, we have

$$
\begin{array}{r}
d\left(h\left(t_{1}\right), h\left(t_{2}\right)\right)<d\left(h\left(s_{1}+\tau_{k-2}\right), h\left(s_{1}\right)\right)+d\left(h\left(s_{1}\right), h\left(s_{2}\right)\right) \\
+d\left(h\left(s_{2}\right), h\left(s_{2}+\tau_{k-2}\right)\right)<\varepsilon
\end{array}
$$

for any $h$ in $\mathcal{H}$, which implies that $\mathcal{H}$ is indeed uniformly equicontinuous on $\mathbb{R}^{+}$. To verify that $\mathcal{H}\left(\mathbb{R}^{+}\right)$is totally bounded in $\mathbb{E}$, we start again from the equicontinuity of $\mathcal{H}$ to obtain a finite cover $\left\{T_{i}\right\}_{i=1}^{n}$ of $[0,3 N]$ and $t_{i} \in$ $T_{i}, i=1, \ldots, n$ such that for every $h \in \mathcal{H}$

$$
d\left(h(t), h\left(t_{i}\right)\right) \leq \frac{\varepsilon}{2} \text { whenever } t \in T_{i}, i=1, \ldots, n .
$$

If $t>3 N$, let us choose $k \in \mathbb{N}$ so that $t \in[k N,(k+1) N]$. Setting $s=$ $t-\tau_{k-2}$, we then have that $s \in[N, 3 N]$ whence $s \in T_{i}$ for some $i \in 1, \ldots, n$. Therefore, given any $h \in \mathcal{H}$, we get

$$
d\left(h(t), h\left(t_{i}\right)\right)<d\left(h\left(s+\tau_{k-2}\right), h(s)\right)+d\left(h(s), h\left(t_{i}\right)\right)<\varepsilon .
$$

Since for each $i=1, \ldots, n$ the set $\mathcal{H}\left(t_{i}\right)$ is totally bounded, we deduce in view of inequality $(6)$ that $\mathcal{H}\left(\mathbb{R}^{+}\right)$is totally bounded.

Theorem 4.2 Let $\mathcal{H}$ be a subset of $\mathcal{C}\left(\mathbb{R}^{+} ; \mathbb{E}\right)$. The following conditions are equivalent:

1. The set $\widetilde{\mathcal{H}}^{+}=\left\{\widetilde{h}(t), h \in \mathcal{H}, t \in \mathbb{R}^{+}\right\}$is a totally bounded subset of $\mathcal{C}_{u}\left(\mathbb{R}^{+} ; \mathbb{E}\right)$ i.e. $\mathcal{H}$ is Bochner-equi-almost periodic.

2. (a) $\mathcal{H}$ is a totally bounded subset in $\mathcal{C}_{k}\left(\mathbb{R}^{+} ; \mathbb{E}\right)$, and

(b) $\mathcal{H}$ satisfies Bochner's uniform double sequence criterion.

3. (a) $\mathcal{H}$ is a totally bounded subset in $\mathcal{C}_{k}\left(\mathbb{R}^{+} ; \mathbb{E}\right)$, and

(b) $\mathcal{H}$ is equinormal.

4. (a) $\mathcal{H}$ is a totally bounded subset in $\mathcal{C}_{k}\left(\mathbb{R}^{+} ; \mathbb{E}\right)$, and

(b) $\mathcal{H}$ is equi-asymptotically almost periodic in Bohr's sense. 
Proof From [6], we have the equivalence between 2 and 3. From [22, Theorem 5, and Theorem 6 page 56] and Lemma 4.1, we deduce the equivalence between 3 and 4 .

There remains to show that 4 is equivalent to 1 .

Assume 4, then in view of Lemma 4.1 the subset $\mathcal{H}\left(\mathbb{R}^{+}\right)$is totally bounded in $\mathbb{E}$, thus $\widetilde{\mathcal{H}}^{+}(t)$ is totally bounded in $\mathbb{E}$ for each $t \in \mathbb{R}^{+}$. We have also in view of Lemma 4.1 that $\widetilde{\mathcal{H}}^{+}$is uniformly equicontinuous. Thus by Ascoli Theorem $\left[8\right.$, Theorem 2 , page X.17], it is totally bounded in $\mathcal{C}_{u}\left(\mathbb{R}^{+} ; \mathbb{E}\right)$.

Conversely, it is obvious that if $\widetilde{\mathcal{H}}^{+}$is a totally bounded subset of $\mathcal{C}_{u}\left(\mathbb{R}^{+} ; \mathbb{E}\right)$ then $\mathcal{H}$ is a totally bounded subset of $\mathcal{C}_{k}\left(\mathbb{R}^{+} ; \mathbb{E}\right)$. There remains to show that $\mathcal{H}$ is equi-asymptotically almost periodic in Bohr's sense. For every $\varepsilon>0$, we can construct a finite cover $\left\{T_{i}\right\}_{i=1}^{n}$ of $\mathbb{R}^{+}$and $t_{i} \in T_{i}, i=1, \ldots, n$ such that, for every $\omega \in \mathbb{R}^{+}$, we have

$$
d\left(h(\omega+t), h\left(\omega+t_{i}\right)\right) \leq \varepsilon, \text { whenever } t \in T_{i}, i=1, \ldots, n .
$$

Let $r=l>\max \left\{t_{1}, t_{2}, \ldots, t_{n}\right\}$, and let us set

$$
\mathcal{A}=\left[\bigcup_{1}^{n}\left(T_{i}-t_{i}\right)\right] \bigcap[r,+\infty[.
$$

Let us check that $\mathcal{A} \cap[t, t+l] \neq \emptyset$ for all $t \geq r$, i.e. that $\mathcal{A}$ is a relatively dense set in $\left[r,+\infty\left[\right.\right.$. Let $t \geq r$, since $\left\{T_{i}\right\}_{i=1}^{n}$ is a cover of $\mathbb{R}^{+}$and $t+l \in \mathbb{R}^{+}$, there exists $i \in 1, \ldots, n$ such that $t+l \in T_{i}$. Observe that $t \leq t+l-t_{i} \leq t+l$ and then $t+l-t_{i} \in \mathcal{A} \cap[t, t+l]$. Now, for a given $t \geq r$ and $\tau \in \mathcal{A}$, let us choose $i \in\{1, \ldots, n\}$ and $s \in T_{i}$ such that $\tau=s-t_{i}$. Since $t-t_{i} \geq 0$ we have, for all $t \in[r,+\infty[$ and for all $h \in \mathcal{H}$

$$
d(h(t+\tau), h(t))=d\left(h\left(t-t_{i}+s\right), h\left(\left(t-t_{i}\right)+t_{i}\right)\right)<\varepsilon .
$$

Hence $\mathcal{H}$ is equi-asymptotically almost periodic in Bohr's sense.

Corollary 4.3 Let $x \in \mathcal{C}\left(\mathbb{R}^{+} ; \mathbb{E}\right)$. The following two statements are equivalent:

1. The set $\widetilde{\mathcal{H}}^{+}(x)=\left\{\widetilde{x}(t), t \in \mathbb{R}^{+}\right\}$is totally bounded in $\mathcal{C}_{u}\left(\mathbb{R}^{+} ; \mathbb{E}\right)$.

2. $x$ is asymptotically almost periodic in Bohr's sense. 
Almost periodicity and asymptotic almost periodicity in completely regular spaces Let $\mathbb{E}$ be a separable completely regular space, let $\mathfrak{U}$ be a uniformity on $\mathbb{E}$ which is compatible with the topology of $\mathbb{E}$, and $\mathfrak{D}$ a familly of semimetrics on $\mathbb{E}$ which induce the uniformity. We denote by $\mathbb{E}_{d}$ the semimetric space $(\mathbb{E} ; d)$.

Lemma 4.4 Let $x$ in $\mathcal{C}(I ; \mathbb{E})$. For each $d \in \mathfrak{D}$, let $x_{d}=\mathrm{i}_{d} \circ x$, where $\mathrm{i}_{d}: \mathbb{E} \rightarrow \mathbb{E}_{d}$ is the canonical injection. Then,

1. $x$ is Bohr-almost periodic (resp. Bohr-asymptotically almost periodic) if and only for each semimetric $d$ in $\mathfrak{D}$, the function $x_{d}$ is Bohr-almost periodic (resp. Bohr-asymptotically almost periodic).

2. $x$ is Bochner-almost periodic if and only for each semimetric $d$ in $\mathfrak{D}$, the function $x_{d}$ is Bochner-almost periodic.

\section{Proof}

1. Assume that $x$ is Bohr-almost periodic. Let $d$ be a semimetric in $\mathfrak{D}$ and let $\varepsilon>0$. The set $V=\{(x, y) \in \mathbb{E} \times \mathbb{E} ; d(x, y)<\varepsilon\}$ is in $\mathfrak{U}$. Since $x$ is almost periodic, there exists a real number $l=l(V)>0$ such that every interval $[a, a+l]$ contains at least one point $\tau$ satisfying

$$
(x(t+\tau), x(t)) \in V, \text { for every } t \in I
$$

hence

$$
d(x(t+\tau), x(t))<\varepsilon, \text { for every } t \in I
$$

which means that $x_{d}$ is almost periodic.

Conversely, assume that for each $d$ in $\mathfrak{D}$, the function $f_{d}$ is almost periodic i.e. for each $\varepsilon>0$ there exists a real number $l=l(\varepsilon, d)>0$ such that every interval $[a, a+l]$ contains at least one point $\tau$ such that

$$
d(x(t+\tau), x(t))<\varepsilon, \text { for every } t \in I .
$$

Let $V$ in $\mathfrak{U}$, then there exists a semimetric $d$ in $\mathfrak{D}$ such that

$$
\{(x, y) ; d(x, y)<\varepsilon\} \subset V .
$$

In particular, we have

$$
(x(t+\tau), x(t)) \in V, \text { for every } t \in I,
$$

and thus $x$ is almost periodic. 
2. In view of Lemma 3.1, the equivalence we wish to prove is a direct consequence of the fact that the set $\{\widetilde{x}(t), t \in I\}$ of translation mappings associated to $x$ is totally bounded in the space $\mathcal{C}_{u}(I ; \mathbb{E})$ if and only if, for each $d \in \mathfrak{D}$, the set $\left\{x_{d}(t+),. t \in I\right\}$ of translation mappings associated to $x_{d}$ is totally bounded in the space $\mathcal{C}_{u}\left(I ; \mathbb{E}_{d}\right)$.

Remark 4.5 By passing to the quotient spaces $\breve{\mathbb{E}}_{d}$, the previous Lemma reduces the study of almost periodicity and asymptotic almost periodicity in uniform spaces to that of almost periodicity and asymptotic almost periodicity in metric spaces. Thanks to Lemma 4.4, we can extend to uniform spaces the equivalence between Bohr-almost periodicity (resp. Bohrasymptotic almost periodicity) and Bochner-almost periodicity when $I=\mathbb{R}$ (resp. $I=\mathbb{R}^{+}$). This result is well known in the case when $I=\mathbb{R}$, see [12]. In the case when $I=\mathbb{R}^{+}$and $\mathbb{E}$ is a locally convex space, it is also well-known, see [17].

Theorem 4.6 Let $\mathbb{E}$ be a uniform space (nonnecessarily complete) and $f$ : $I \rightarrow \mathbb{E}$ a continuous function. Then

1. If $I=\mathbb{R}$, the following statements are equivalent:

(a) $x$ is Bohr-almost periodic.

(b) $x$ is Bochner-almost periodic.

2. If $I=\mathbb{R}^{+}$, the following conditions are equivalent:

(a) $x$ is Bohr-asymptotically almost periodic.

(b) $x$ is Bochner-almost periodic.

Proof This is a direct consequence of Lemma 4.4 and of the corresponding result in (semi)metric spaces.

Aknowledgements We heartily thank François Charlot for pointing out with a counterexample (not included here) some errors in a previous version of this work. We are also indebted to Thierry de la Rue for Counterexample 2.16 . 


\section{References}

\section{References}

[1] L. Arnold and C. Tudor. Stationary and almost periodic solutions of almost periodic affine stochastic differential equations. Stochastics Stochastics Rep., 64:177-193, 1998.

[2] Paul H. Bezandry and Toka Diagana. Existence of almost periodic solutions to some stochastic differential equations. Appl. Anal., 86(7):819827, 2007.

[3] Paul H. Bezandry and Toka Diagana. Square-mean almost periodic solutions nonautonomous stochastic differential equations. Electron. J. Differential Equations, pages No. 117, 10 pp. (electronic), 2007.

[4] Paul H. Bezandry and Toka Diagana. Existence of quadratic-mean almost periodic solutions to some stochastic hyperbolic differential equations. Electron. J. Differential Equations, pages No. 111, 14, 2009.

[5] Patrick Billingsley. Convergence of probability measures. Wiley Series in Probability and Statistics: Probability and Statistics. John Wiley \& Sons Inc., New York, second edition, 1999. A Wiley-Interscience Publication.

[6] S. Bochner. A new approach to almost periodicity. Proc. Nat. Acad. Sci. U.S.A., 48:2039-2043, 1962.

[7] N. Bourbaki. Éléments de mathématique. Topologie générale. Chapitres 1 à 4. Hermann, Paris, 1971.

[8] Nicolas Bourbaki. General topology. Chapters 5-10. Elements of Mathematics (Berlin). Springer-Verlag, Berlin, 1998. Translated from the French, Reprint of the 1989 English translation.

[9] G. Da Prato and C. Tudor. Periodic and almost periodic solutions for semilinear stochastic equations. Stochastic Anal. Appl., 13(1):13-33, 1995.

[10] Thierry de la Rue. Mouvement moyen et système dynamique gaussien. Probab. Theory Related Fields, 102(1):45-56, 1995. 
[11] Ryszard Engelking. General topology, volume 6 of Sigma Series in Pure Mathematics. Heldermann Verlag, Berlin, second edition, 1989. Translated from the Polish by the author.

[12] N. Gheorghiu. Deux définitions des fonctions presque-périodiques continues à valeurs dans un espace vectoriel topologique. An. Şti. Univ. "Al. I. Cuza" Iaşi Secţ. I a Mat. (N.S.), 15:329-334, 1969.

[13] T. Morozan and C. Tudor. Almost periodic solutions to affine Ito equations. Stoch. Anal. Appl., 7(4):451-474, 1989.

[14] Octav Onicescu and Vasile I. Istrătescu. Approximation theorems for random functions. Rend. Mat. (6), 8:65-81, 1975. Collection of articles dedicated to Mauro Picone on the occasion of his ninetieth birthday.

[15] A. M. Precupanu. On the almost periodic functions in probability. Rend. Mat. (7), 2(3):613-626, 1982.

[16] W. M. Ruess and W. H. Summers. Asymptotic almost periodicity and motions of semigroups of operators. In Proceedings of the symposium on operator theory (Athens, 1985), volume 84, pages 335-351, 1986.

[17] W. M. Ruess and W. H. Summers. Compactness in spaces of vector valued continuous functions and asymptotic almost periodicity. Math. Nachr., 135:7-33, 1988.

[18] Laurent Schwartz. Radon measures on arbitrary topological spaces and cylindrical measures. Published for the Tata Institute of Fundamental Research, Bombay by Oxford University Press, London, 1973. Tata Institute of Fundamental Research Studies in Mathematics, No. 6.

[19] C. Tudor. Almost periodic solutions of affine stochastic evolution equations. Stochastics Stochastics Rep., 38(4):251-266, 1992.

[20] C. Tudor. Almost periodic stochastic processes. In Qualitative problems for differential equations and control theory, pages 289-300. World Sci. Publ., River Edge, NJ, 1995.

[21] Ward Whitt. Weak convergence of probability measures on the function space $C[0, \infty)$. Ann. Math. Statist., 41:939-944, 1970.

[22] S. Zaidman. Almost-periodic functions in abstract spaces, volume 126 of Research Notes in Mathematics. Pitman (Advanced Publishing Program), Boston, MA, 1985. 